\title{
Transthoracic Real-Time Three-Dimensional Echocardiography Using the Rotational Scanning Approach for Data Acquisition
}

\author{
ACHI LUDOMIRSKY, ROGER VERMILION, JOHACHIM NESSER, GERALD MARX, \\ MICHAEL VOGEL, RICHARD DERMAN, and NATESA PANDIAN \\ University of Michigan, Ann Arbor, Michigan; and Tufts-New England Medical Center, \\ Boston, Massachusettes
}

Transthoracic real-time three-dimensional echocardiography using the rotational scanning approach for data acquisition became a feasible modality for cardiac imaging during the last 4 years. Several attempts for reconstruction of the heart have been made using different methods. In this study we evaluate the data acquisition using the rotational approach from the transthoracic window. Thirty-five children with congenital heart disease were enrolled in the study. All of them underwent complete two-dimensional and Doppler echocardiogram followed by three-dimensional reconstruction using rotational image acquisition. The rotational approach enabled us to acquire good data from subcostal, apical, and suprasternal notch positions by rotating the transducer from $0^{\circ}-180^{\circ}$. Novel views of the atrioventricular valve, semilunar valve, atrial, and ventricular septi were established by spatial plane imaging from the three-dimensional echocardiography. This reconstruction enables spatial imaging of cardiac structures and deficiencies. Three-dimensional echocardiography will enhance the understanding of complex congenital heart disease. (ECHOCARDIOGRAPHY, Volume 11, November 1994)

congenital heart disease, three-dimensional echocardiography, four-dimensional echocardiography, rotational approach, image processing

Three-dimensional reconstruction is widely used in the field of radiology by means of CT and MRI. From the tomographic slices threedimensional reconstruction is done either mentally in the brain of the physician or via hardware and software using a dedicated three-dimensional work station. ${ }^{1}$ The information from parallel slices on a multiformat display and/or three-dimensional surface rendering display is important for the diagnosis to be made based upon these modalities. Although three-dimensional data has been used to perform a limited range of measurements from endocardial borders, cardiac ultrasound until now has not offerred three-dimensional imaging information

Address for correspondence and reprints: Achi Ludomirsky, M.D., Pediatric Cardiology, University of Michigan, F1541 Mott, Box 0204, 1500 East Medical Center Drive, Ann Arbor, MI 48109. Fax: 313-936-9470. for the importance feature. During the last 2 years, however, three-dimensional echocardiography had evolved as a feasible modality for cardiac imaging. Several attempts for reconstruction of the heart have been made using different methods. ${ }^{2-4}$

Several problems arose for data acquisition process including the ability to reconstruct a structure acquiring adequate images. Three main approaches were suggested for data acquisition: (1) parallel scanning; (2) pyramidal; or (3) rotational..$^{5-8}$ We studied the use of rotational scanning reconstruction in children and adults with congenital heart disease. The rotational approach enabled us to acquire good datasets from the subcostal position (especially in children) as well as from the parasternal window, by rotating the transducer from $0^{\circ}$ to $180^{\circ}$.

Many investigators are using the transesophageal window with a rotational or parallel 
approach for data acquisition. With the evolving multiplane capabilities of these transducers, rotational (rotoplane), echo CT was developed in order to collect good image datasets, and to transfer and store them within the process of three-dimensional reconstruction. In order to obtain adequate four-dimensional datasets all of these techniques require precise spatial localization of image plane, as well as phasic temporal resolution using excellent ECG respiration and gating. In this study we present our initial experience with the rotational scan reconstruction acquisition strategy for transthoracic four-dimensional real-time echocardiographic studies in the pediatric and adult patients with congenital heart disease.

\section{Method}

\section{Instrumentation and Data Acquisition}

We used commercially available echocardiographic imaging (Interspec RX400, Ambler, PA, USA) interfaced with a dedicated three-dimensional imaging processing computer unit (Echoscan Tomtec, Inc, Denver, CO, USA). A symmetrical focus transthoracic ultrasound transducer probe (5-and $7.5-\mathrm{MHz}$ frequency when used on children and $3.5 \mathrm{MHz}$ frequency transducer when used on adults) was mounted and centered in a double walled cylindrical rotation apparatus.

The outside wall of the rotating apparatus was mounted on the distal end of a modified mechanical arm assembly, which was in turn mounted on the Apogee CX200 echocardiographic machine. The probe was easily positioned on the patient in an optimal subcostal or parasternal windows and locked into place. The inner tube of the rotating apparatus was attached to the ultrasound probe with a centering mechanism. The tube was able to rotate freely and concentrically to the outer wall. The rotation was controllable by the Echoscan system via a miniature motor.

The dataset collection was controlled and acquired by the echo CT system. Before data collection began the system determined the proper number of slices that compromise the rotational dataset. Based on the patient's heart rate and available system memory (approximately 60 megabytes). The slices must be evenly distributed over $180^{\circ}$ required for the rotated dataset. For our patients there were 180 slices. Critical placement on the patient's chest of a three-lead set provides both ECG and respiration information during data acquisition. Real-time echo video from the subcostal and/or parasternal located probe was fed into the echo CT system. Sophisticated algorithm based on ECG and respiration determined whether a given heartbeat was valid. Echo data from valid heartbeats were stored. Immediately after the heartbeat was determined to be valid the probe was step rotated by one slice thickness. The rotation step was rapid and small enough to assure that all video frames in a slice come from the same tomographical location. Once the scanning sequence is completed the digital images were stored into the echo CT memory. The result was a series of conical datasets of the cardiac anatomy over one cardiac cycle that was able to be analyzed and processed. Annular array probes were chosen for the transthoracic three-dimensional data acquisition because they are ideal in both form and acoustics. The hemispherical nose of the probe provides acoustic footprint that does not change as the probe is rotated. The probe can easily glide on a thin layer of couplant during the acquisition. More importantly, however, is the symmetrical beam focusing of the annular array. This permits both in plane (azimuthal) and out of plane (elevational) focusing. Without elevational focusing there would be the potential significant loss of elevation resolution resulting in highly anisotropic datasets with certain viewing angles and slices yielding poorer data than others.

\section{Data Processing}

Processing of the dataset was performed off line by the Echoscan as described previously by several authors. ${ }^{9 \cdot 12}$ The conical datasets were digitally reformatted and numerous new view planes were utilized to process and create a different look at the anatomical details. The capability of cutting the dataset in any plane provided three-dimensional imaging along these cut planes. Establishing a cut plane in the $\mathrm{X}, \mathrm{Y}$, and 
$\mathrm{Z}$ coordinate was important before further processing of the data. Once the desired cut plane was found, which showed the desired analconical segmentation was performed and three-dimensional images were obtained using multiple simultaneous rendering algorithms. The different renderings then could be mixed to achieve a final result. Surface and volume rendering techniques were used in all cases to enable one to change the gray values and texture shading.

\section{Patient Population}

We studied 35 consecutive patients with congenital heart disease. Age ranged from 3 months to 19 years with a mean of 7.2 years. Patients' pathology consists of hypoplastic left heart, tricuspid atresia, atrial and ventricular septal defect, aortic or pulmonary stenosis, atrioventricular canal, coarctation of the aorta, Ebstein's anomaly, double outlet right ventricle, and double inlet left ventricle. All children below 4 years of age were sedated. A total of 42 rotational scans were performed from different echocardiographic views: 14 subcostal, 14 apical, 12 parasternal, and 2 right subclavian.

\section{Results}

Adequate data collection was possible in all 35 patients. A total of 42 scanning sequences were stored and analyzed. Average image acquisition time was 1.8 minutes (depending on the heart rate and the patient mobility) and ranged between 1-3.4 minutes. All but two studies resulted in successful diagnostic quality reconstructions that could be formatted, reviewed, and rendered into three-dimensional moving dynamic images. Time for a three-dimensional rendering varied from 15 minutes for the first low level resolution to up to 2-3 hours for a final multiperspective rendering ( 1 pixel level).

Previous unobtainable cut planes used in different orientations yielded unique and useful intracardiac views for patients with congenital heart disease before and after repair. It was to our surprise that in the five adults with congenital heart disease in which only a parasternal rotation provide adequate two-di- mensional images, three-dimensional reconstruction was successful.

Three-dimensional images derived from data obtained in the parasternal view enabled us to evaluate cardiac anatomy in the conventional long-and short-axis parasternal views (Fig. 1). A close look at the mitral and aortic valve and evaluation of the mitral aortic continuity is possible. Assessment of left atrial size and configuration and mitral apparatus including supravalvar area, annulus, mitral valve leaflet, chordi, and papillary muscle was also possible from this approach. Imaging of the mitral valve was possible from both the left atrial and left ventricular approach. Both gave an excellent differentiation and delineation of the posterior medial and anterior lateral leaflets. In the dynamic form with moving imaging a sense of rapid filling and atrial kick of the left atrium was easily depicted. Evaluation of mitral valve prolapse into the left atrium as seen from the left atrial approach are easily obtained. In the case of mitral insufficiency because of a lack of coaptation of the mitral leaflets is seen from the left ventricular approach aiming anterior and superior (Fig. 2). The parasternal approach also gave adequate information of the right ventricular inflow. Three leaflets of the tricuspid valve were easily seen and the septal attachment of the septal leaflet to the ventricular septum were also demonstrated.

Imaging of the aortic valve was achieved from both the supraaortic valve area and from

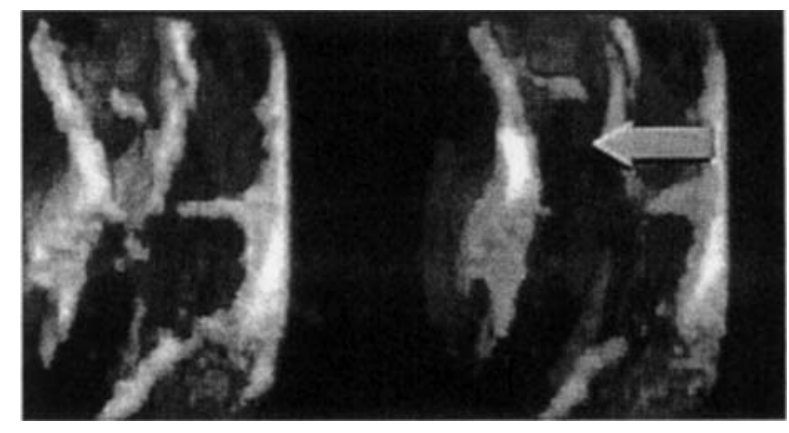

Figure 1. Three-dimensional echocardiographic reconstruction of normal heart in a 38-year-old patient. The left atrium, mitral valve, left ventricular outflow tract, and aortic valve are well demonstrated. 


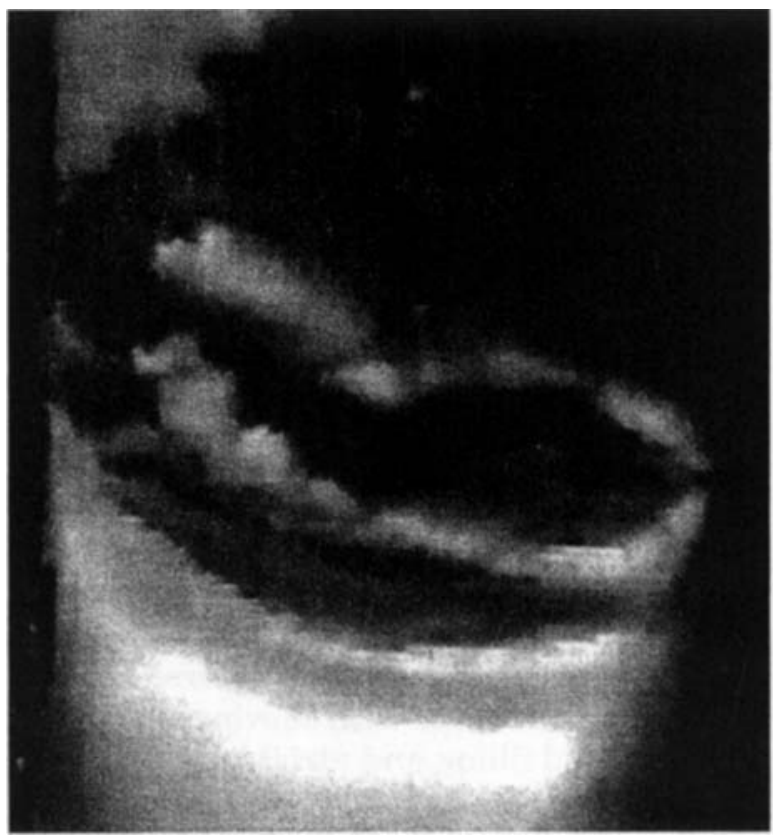

Figure 2. Three-dimensional echocardiographic reconstruction of the mitral valve in a patient with moderate mitral regurgitation. The view is from the left ventricle toward the left atrium. Note the lack of coaptation of the mitral leaflets in systole.

the left ventricular outflow tract region imaging from inferior to superior. A novel orientation of the aortic valve view from the ascending aorta easily depicted tricuspid versus bicuspid valve conditions, thickening of the valve leaflet, opening of the valve, and the presence of any subaortic obstruction or endocarditis (Fig. 3). Rotating this view on the $\mathrm{X}$ or $\mathrm{Y}$ axis enabled easy demonstration of the proximal coronary arteries origin within the sinuses and up to two centimeters in distance from takeoff. Rotation of the heart around the $\mathrm{X}$ axis from this plane displayed a real time of dynamic cardiac anatomy showing the left ventricular apex and the base of the papillary muscle.

Subcostal rotation using annular array probes provided sufficient and excellent two-dimensional images in all children with congenital heart disease yielding to excellent three-dimensional reconstruction of the heart including the inflow and outflow tracts. A conventional four-chamber view in three-dimensional display serves as the basic image for reconstruc- tion. Right and left atrium are easily seen and the atrial septum is displayed in several projections delineating the convex-concave characteristic of these structures and depicting atrial septal defects in an accurate spatial location.

In patients with atrial septal defect and atrioventricular septal defect size, location, and spatial orientation of the atrial septal defects were obtained by using the "surgical" view either from the left atrium or right atrium, respectively. The borders of the atrial septal defect and the existence of strands or fenestrated atrial septal defects were easily seen by this technique (Fig. 4). Atrial septal defect size as changing during systole and diastole are easily seen in this projection. Pulmonary vein and left atrial appendage were depicted especially in the real-time display when the heart was rotated on the $\mathrm{Y}$ axis (Fig. 5). The left upper pulmonary vein and left atrial appendage were easily displayed and the contractile characteristic of the left atrial appendage was more appreciated with this projection. Reconstruction of the atrioventricular valves from both the atrial and the ventricular projection were possible and en-

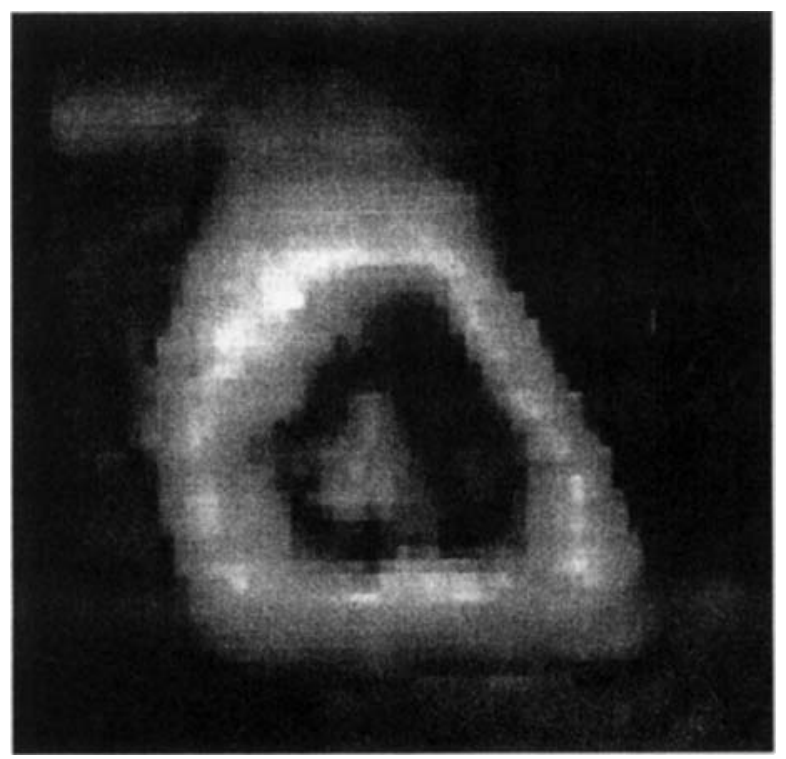

Figure 3. Three-dimensional echocardiographic reconstruction of the aortic valve from the ascending aorta looking down toward the left ventricular outflow tract. The aortic valve cusps are thickened and there is limited leaflet excursion. 


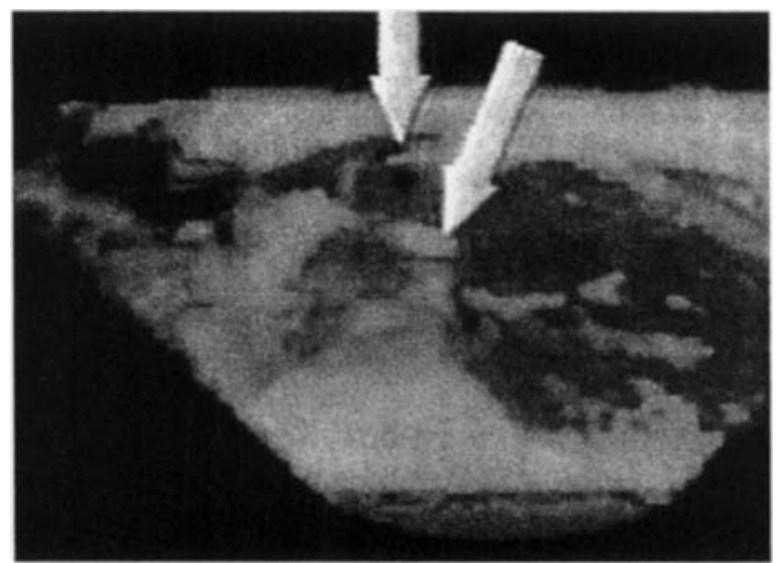

Figure 4. Three-dimensional echocardiographic reconstruction of the atrial septum in a patient with an atrial septal defect. A novel orientation looking from the right atrium into the left atrium. The atrial septal defect borders are well seen.

hance the visual characteristics of these valves. The ventricular septum along its muscular, perimembranous, and membranous areas was reconstructed and displayed from the conventional four-chamber view as well as displayed from the right and left ventricular cavities, respectively. In a case of one ventricular septal defect repair with a large patch, the attachment of the patch to the ventricular septum along its borders were easily obtained using a three-dimensional reconstruction from the right ventricular projection. Patient pericardial effusion was easily detected and the extension of the effusion was nicely projected using the three-dimensional reconstruction from the rotational transthoracic approach. The extension of the ef-

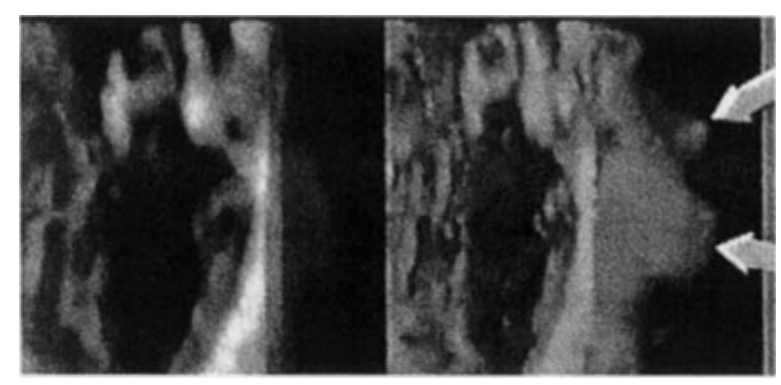

Figure 5. Three-dimensional echocardiographic reconstruction of the left ventricle after rotation across the Y-axis. The left atrial appendage and left upper pulmonary vein are well seen. fusion can be easily seen from the posterior and anterior projection surrounding the heart and localization of the effusion is easily detected (Fig. 6). In three cases with Ebstein's anomaly the anatomic details of the right ventricle were accessed by three-dimensional images. The atrialized portion of the right ventricle was delineated and the true and new annuli of the rricuspid valve were visualized. These images were possible using a novel view plane in which the right and left atrial roofs were electronically removed and a look from the atria into the ventricles was possible. The amount of valve tissue, the orientation, and the degree of displacement of the right atrioventricular valve were evaluated in this projection.

\section{Discussion}

This data demonstrates that dynamic transthoracic three-dimensional echocardiography is feasible using rotational scanning method for data acquisition. It is encouraging to realize that not only the subcostal view in small children enabled us to acquire good images and adequate three-dimensional reconstruction but the parasternal approach in children as well as in adults provides essential and

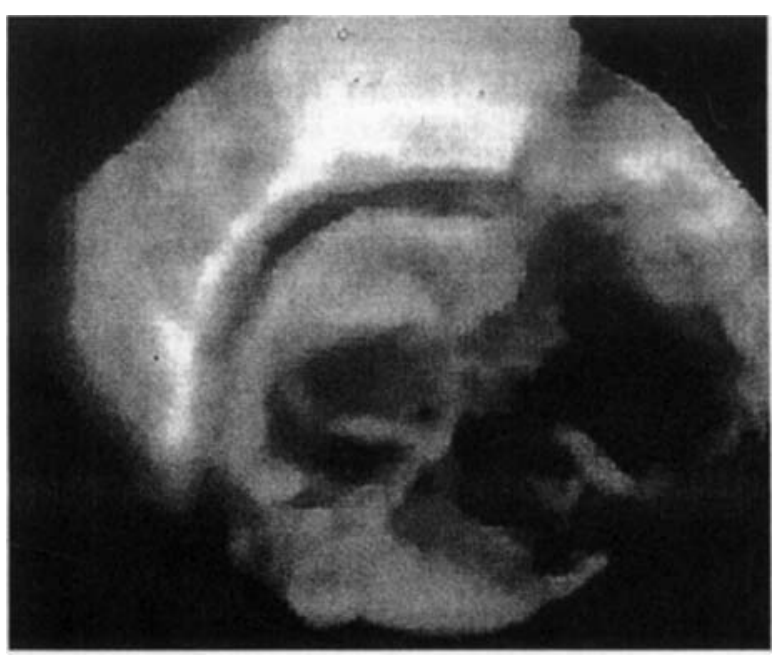

Figure 6. Three-dimensional echocardiographic reconstruction of a patient with hypoplastic left heart syndrome, status post Norwood operation. There is circumferential pericardial effusion. The extension of the effusion is well seen. 
new information using three-dimensional echocardiography. Three-dimensional imaging of the heart by ultrasound has been attempted for many years and several methods have been proposed. ${ }^{13-16}$ There are several main concerns and problems that followed this process: (1) The time consuming process of coordinating cardiac cross section with the spatial location; and (2) the image quality of the reconstruction.

In the past year three-dimensional reconstruction using ultrasound data became feasible, which allows a display of both the surface of the structure and shading of the inside of the cardiac chambers. In this study, we used a new three-dimensional visualization technique that used different rendering algorithms that apply to this reconstruction.

Various methodologies were used in order to project spatial information incorporated into the three dataset.

(1) Surface modeling: In this technique surface contour of the object must be extracted. These contours can then be used for a polygram based or triangulated three-dimensional display. The disadvantage of this method is that only the surface of this object can be displayed. A cut plane applied through three-dimensional display would not show the three-dimensional information inside the object.

(2) Surface rendering: This allowed us to display either the surface of the object with a shading of the interior parts of the volume with its gray value or both together. This technique can display three-dimensional data directly from the gray scale volumes. In the surface rendering technique the three-dimensional object can be divided into two main steps: (1) definition of the object boundaries; and (2) the calculation of the shaded presentation of the object. In the definition of the object boundary along each array a search for the object surface is performed: usually a gray level threshold is used to separate an object from the background. Gray value above the threshold defined the object. Gray value below the object defined the background. When calculating the shaded presentation of the object the five different rendering algorithms were used. The result of the recon- struction can then be mixed together with different weighting factors. This allowed us to utilize the advantage of each algorithm in the resulting three-dimensional reconstruction.

The five different algorithms included:

(1) The distant shading: In this modality the distance from the observer to the surface of the object is converted into a gray value. Surface points that are more distant to the observer will become darker than points that are close to the observer. This shading method gives rather smooth views of the object with very few structural details.

(2) Gradient shading: A realistic illumination model is used. Light is needed and is reflected at the source of the object. The light source is assumed to be mounted on the the observor's head. The surface normal needed for the determination of the direction of the reflected light is calculated from the gray level gradient of the object surface. Gradient shading leads to very realistic and detailed views of the object but it is highly susceptible to artifact.

(3) Texture: In this context texture means the representative of gray level of the object taken at the object surface. Thus the characteristic appearance of ultrasound images can be retained and added to the distance of gradient or gradient shaded representation of the object.

(4) Maximum/minimum modality is gray value along each ray through which the data volume is displayed. The result is anquasitransparent views of the object where a bright versus dark structure is represented. The depth perception in single images are rather poor, but it can be greatly appreciated and enhanced when rotating sequences are calculated.

In this study we explored the utility of the transthoracic rotational approach for imaging of congenital heart disease in children and adults. The novel views of the atrioventricular valve and semilunar valve from the "surgeon" approach are feasible and open a new window in cardiac imaging. The surgeon can practically design and plan the procedure in an environment other than the operating room. Threedimensional echocardiographic reconstruction from the transthoracic approach enabled spa- 
tial imaging of cardiac structures and deficiencies. In the case of atrial septal and ventricular septal defect the relation between the extension of the defect, the atrioventricular valves, the roof of the atrial septum, as well as the involvement of different parts of the ventricular septum was more appreciated from the new surgical views within the left and right atrium and ventricles. In patients who are candidates for Rastelli operation, the knowledge of exact size of the ventricular septal defect is crucial for a successful operation including implementation of intra-and extracardiac conduits. Visualization of the atrial septal defect from the right atrium will give a new insight of the atrial septal defect morphology and will give the interventional catheterizers a new insight into the possibility of closing these holes with transcatheter devices. ${ }^{17,18}$

Three-dimensional echocardiography can be used as a superb teaching tool for medical students, residents, and fellows especially in the field of pediatric cardiology and congenital heart disease. We believe that the capability of slicing and visualization of the heart in practically unlimited views will enhance the understanding of complex congenital heart disease.

\section{Limitations}

Acquisition time ranged between 1-3.4 minutes with a mean of 2.3 depending on the heart rate and the mobility of the patient. In the pediatric age group, especially in patients under 4 years of age, sedation is needed in order to acquire adequate images. The reconstruction is a long process and a final high quality image can be achieved after 3 hours. This is a very long period even though the first low resolution four pixel three-dimensional reconstruction could be achieved within 15 minutes. With improvement of computer power we expect this problem to be eliminated. In several of our data acquisitions a motion artifact or a rotation artifact was noted in the middle of the display. This resulted from the rotational approach and can be minimized by better angulation and mounting of the ultrasound probes within the automatic rotational arm.
There is a relatively long learning curve for three-dimensional reconstruction. Nonconventional views are being produced and in patients with complex heterotaxy congenital heart syndrome, the orientation could be confused in several cases. We recommend that a careful two-dimensional evaluation should be performed before the three-dimensional reconstruction is acquired. Once the dataset is in place the operator should be able to choose the best views from which a three-dimensional reconstruction is derived.

\section{Future Implication}

At present three-dimensional reconstruction after acquisition of the basic data is still time consuming. Cutting through the volume of data that allowed a display of two-dimensional cross sections in various orientations should reach a point of being less operator dependent, which will decrease the reconstruction time. Also, new developments in hardware, software, and computer power should facilitate the three-dimensional reconstruction and the implementation of new and powerful computers should minimize the time and may bring three-dimensional reconstruction as an online technique. Displays of volume flows in three dimensions, especially regurgitant volume, would enhance our diagnostic capability for regurgitant fraction and assessment of incompetent valves. It should be possible to use color flow displays, acquire this data in cubical form, and perform three-dimensional reconstruction. Other areas that this technique can help will be in the evaluation of regional and global ventricular function and geometrical structure of left and right ventricles.

\section{Conclusion}

In this study we demonstrate that rotational scanning from the transthoracic approach yields an adequate three-dimensional realtime echocardiographic reconstruction. The rotational views allows utilization of arrays of images for reconstruction of specific areas that are preselected from an optimal single points window to demonstrate cardiac structures. The 
ability to focus on areas of specific diagnostic importance through the unique rotational windows makes this tectnique feasible in young pediatric patients and adults with congenital heart disease. The ease of transthoracic acquisition with this new combined system should bring three-dimensional reconstruction closer to clinical application for detailing the complex spatial anatomical relation present in congenital heart disease.

\section{References}

1. Wehrli FW: Principle of Magnetic Resonance in MRI. Stark and Bradely (eds), Toronto, The CV Mosby Publishing, 1980, pp. 3-23.

2. Ghosh A, Nanda NC, Maurer G: Three-dimensional reconstruction of echocardiographic images using the rotation method. Ultrasound Med Biol 1982;6:655-661.

3. Pandian NG, Nanda NC, Schwarts SL, et al: Three-dimensional and four-dimensional transesophageal echocardiographic imaging of the heart and aorta in humans using a computed tomographic imaging probe. Echocardiography 1992;9:677-687.

4. Pini R, Monnini E, Masotti L, et al: Echocardiographic three-dimensional visualization of the heart. NATO ASI Series 1990;60:263-274.

5. Belohlavek M, Foley DA, Gerber TC, et al: Three-and four-dimensional cardiovascular ultrasound imaging: A new era for echocardiography. Mayo Clin Proc 1993;68:221-240.

6. Flachskamp FA, Handschumacher M, Vandervoort PM, et al: Dynamic, three-dimensional reconstruction of the mitral annulus using a multiplane transesophageal echo-transducer. Circulation 1991;84:11-686.

7. Cao QL, Esakof D, Vannan MA, et al: Three-dimensional echocardiography using an onmiplane transesophageal echocardiographic probe: Automated data collection and combined volume and surface rendering allow qualitative and quantitative assessment of cardiac structures and abnormalities (abstract). JACC 1993;21:309A.

8. Pandian NG, Cao QL, Erbel R, et al: A comprehensive approach for image segmentation, cutting planes and display projections in three-dimensional echocardiography: Suggested guidelines for clinically useful projections based on multicenter experience in 300 adult and pedi- atric patients. JACC (In press).

9. King DL, King DL Jr, Shao MY: Three-dimensional spatial registration and interactive display of position and orientation of realtime ultrasound images. $J$ Ultrasound Med 1990;9: 525-532.

10. Schwartz SL, Cao QL, Azevedo J, et al: Simulation of intraoperative visualization of cardiac structures and study of dynamic surgical anatomy with real-time three-dimensional echocardiography in patients. Am $J$ Cardiol 1994;73:501-507.

11. Fulton DR, Marx GR, Pandian NG, et al: Dynamic three-dimensional echocardiographic imaging of congenital heart defect in infants and children by computer-controlled tomographic parallel slicing using a single integrated ultrasound instrument. Echocandiography 1994;11: 155-165.

12. Vogel M, Pandian N, Marx G, et al: Transthoracic real-time three-dimensional echocardiography in 100 pediatric and adult patients with heart disease: Clinical utility of unique new views unavailable in 2-dimensional echocardiography (abstract). Circulation 1993;88:1868.

13. Dekker DL, Piziali R, Dong E: A system for ultrasonically imaging the human heart in three dimensions. Comput Biomed Res 1974;7: 544-553.

14. Matsumoto M, Matsuo H, Kitabatake A, et al: Three-dimensional echocardiograms and twodimensional echocardiographic images at desired planes by a computerized system. Ultrasound Med Biol 1977;3:163-178.

15. Greenlleaf JF: Three-dimensional imaging in ultrasound. J Med Syst 1982;6:579-589.

16. Nikravesh PE, Skorton DJ, Chandran KB, et al: Computerized three-dimensional finite element reconstruction of the left ventricle from cross-sectional echocardiograms. Ultrasonic Imaging 1984;6:48-59.

17. Belohlavek M, Foley DA, Gerber TC, et al: Three-dimensional ultrasound imaging of the atrial septum: Normal and pathologic anatomy. JACC 1993;22:1673-1678.

18. Cao QL, Azevedo J, Marx G, et al: Transthoracic three-and four-dimensional echocardiography in children and adults using a computerized tomographic ultrasound probe: Gray level gradient shading enhances the recognition of intra-cardiac structures and congenital heart defects (abstract). JACC 1993;21:368A. 